\title{
Information propagation for interacting-particle systems
}

\author{
Norbert Schuch, ${ }^{1}$ Sarah K. Harrison, ${ }^{2}$ Tobias J. Osborne, ${ }^{3,4}$ and Jens Eisert ${ }^{4,5}$ \\ ${ }^{1}$ Institute for Quantum Information, California Institute of Technology, MC 305-16, Pasadena, California 91125, USA \\ ${ }^{2}$ Department of Mathematics, Royal Holloway University of London, Egham, Surrey, TW20 OEX, United Kingdome \\ ${ }^{3}$ Institut für Theoretische Physik, Leibniz-Universität Hannover, Appelstrasse 2, DE-30167 Hannover, Germany \\ ${ }^{4}$ Institute for Advanced Study Berlin, DE-14193 Berlin, Germany \\ ${ }^{5}$ Institute of Physics and Astronomy, University of Potsdam, DE-14476 Potsdam, Germany
}

(Received 31 January 2011; published 8 September 2011)

\begin{abstract}
We study the speed at which information propagates through systems of interacting quantum particles moving on a regular lattice and show that for a certain class of initial conditions there exists a maximum speed of sound at which information can propagate. Our argument applies equally to quantum spins, bosons such as in the Bose-Hubbard model, fermions, anyons, and general mixtures thereof, on arbitrary lattices of any dimension. It also pertains to dissipative dynamics on the lattice, and generalizes to the continuum for quantum fields. Our result can be seen as an analog of the Lieb-Robinson bound for strongly correlated models.
\end{abstract}

DOI: $10.1103 /$ PhysRevA.84.032309

PACS number(s): 03.67.Hk, 05.50.+q

\section{INTRODUCTION}

How fast can information propagate through a system of interacting particles? The obvious answer-no faster than the speed of light-is certainly correct; however, it is not the answer one is usually looking for. For instance, in a classical solid, liquid, or gas, perturbations propagate at the speed of sound, which is determined by the way the particles in the system locally interact with each other, without any reference to relativistic effects. We would like to understand whether a similar "speed of sound" exists for quantum interacting-particle systems, limiting the propagation speed of localized excitations, i.e., quasiparticles. For interacting quantum spin systems such a maximal velocity, known as the Lieb-Robinson bound [1-4], has indeed been shown. While it seems reasonable that there should always be such a bound, systems of interacting bosons can exhibit counterintuitive effects, in particular since the interpretation of excitations in terms of particles is no longer fully justified; in fact, an example of a nonrelativistic system where bosons condense into a dynamical state that steadily accelerates has recently been constructed [5]. This example suggests the possibility that our intuition is wrong, and only relativistic quantum theory can provide a rigorous speed limit for bosonic systems.

There are many important reasons to investigate bounds on the speed of information propagation in interacting-particle systems. For instance, such bounds lead directly to important, general results concerning the clustering of correlations in equilibrium states [2], and the mere existence of a LiebRobinson bound for a quantum system can be used to develop efficient numerical procedures to simulate the dynamics of lattice models [6]. From a more practical perspective, new experiments allow one to explore the nonequilibrium dynamics of ultracold strongly correlated quantum particles-bosonic, fermionic, or mixtures thereof-in optical lattices with unprecedented control $[7,8]$. In such experiments, it is important to understand how the particles move. For example, when studying instances of anomalous expansion, it is far from clear a priori whether it is possible to identify a meaningful speed of sound at all.
The original Lieb-Robinson bound applies in a very general setting, namely, to any lattice system with bounded local Hilbert space dimension, such as low-dimensional quantum spin systems, fermionic lattice systems, or hard-core bosons. Equally, Lieb-Robinson bounds can be derived for bosonic systems with quadratic interactions, such as coupled harmonic oscillators, and they remain valid for certain small nonquadratic perturbation bounds, such as small nonharmonic potentials [9]. Yet, this does not cover systems of (strongly) interacting bosons such as the Bose-Hubbard model, which are of great practical interest. Unfortunately, previous attempts to extend the original Lieb-Robinson argument to bosonic systems have run into insuperable difficulties, which are fundamentally connected to the unboundedness of the creation operator for bosons: the Lieb-Robinson velocity depends on the norm of the interaction, which is unbounded for, e.g., bosons hopping on a lattice, and examples without a speed limit have indeed been constructed [5].

In this paper, we show how to prove bounds on the speed of information propagation for a wide class of systems, including the Bose-Hubbard model, under certain conditions on the initial state. In particular, we focus on the scenario where most of the particles are initially confined to a certain region, and study how they propagate into the initially unoccupied region once the confining potential is removed. For instance, this covers the scenario of bosons in a trap, when the trapping potential is turned off, and the bosons expand into the initially unoccupied region.

Our argument applies not only to systems of interacting bosons, but also to fermions, spins, anyons, or mixtures thereof, both on lattices and in the continuum. Moreover, it can also be applied beyond Hamiltonian evolution, such as to systems evolving under some local dissipative dynamics. It should be noted, however, that this does not cover scenarios such as the propagation of perturbations of the equilibrium state of the Bose-Hubbard model inside an optical lattice, which has a macroscopic occupation number in the whole region of interest. 


\section{SETUP}

The type of system we have in mind is exemplified by the Bose-Hubbard model, a model of bosons hopping on an arbitrary lattice $G$ of any finite dimension and interacting via an on-site repulsion,

$$
\begin{aligned}
\hat{H}_{\mathrm{BH}}= & -\tau \sum_{\langle j, k\rangle}\left(\hat{b}_{j}^{\dagger} \hat{b}_{k}+\text { H.c. }\right) \\
& +\frac{U}{2} \sum_{j} \hat{n}_{j}\left(\hat{n}_{j}-1\right)-\mu \sum_{j} \hat{n}_{j},
\end{aligned}
$$

where the first summation is over neighboring sites on the lattice, $\hat{b}_{j}$ is the boson annihilation operator for site $j$, and $\hat{n}_{j}=\hat{b}_{j}^{\dagger} \hat{b}_{j}$ is the number operator. The natural distance in the lattice will be denoted by $d(\cdot, \cdot)$, e.g., $d(j, k)=|j-k|$ for a one-dimensional chain. While we, for clarity, focus our discussion on the Bose-Hubbard model, our arguments directly generalize to models of the form

$$
\hat{H}=-\tau \sum_{s=1}^{S} \sum_{\langle j, k\rangle}\left(\hat{b}_{s, j}^{\dagger} \hat{b}_{s, k}+\text { H.c. }\right)+f\left(\left\{\hat{n}_{1, j}, \ldots, \hat{n}_{S, j}\right\}_{j \in G}\right),
$$

where the $\hat{b}_{s, j}$ are annihilation operators for bosons, fermions, or even anyons of species $s=1, \ldots, S$ at site $j$, and $\hat{n}_{s, j}=$ $\hat{b}_{s, j}^{\dagger} \hat{b}_{s, j}$; the species could for instance refer to an internal spin degree of freedom. The interaction between the particles is characterized by $f$, which can be an arbitrary function of the local densities, and may involve higher moments of the particle number, or even nonlocal interactions. Moreover, our argument also applies to time-dependent Hamiltonians of this form, as long as the tunneling amplitude $\tau(\cdot)$ is bounded.

The scenario we consider (see Fig. 1) is described by the Bose-Hubbard model on a lattice $G$, where in the initial state all sites are empty (i.e., $\left\langle\hat{n}_{j}\right\rangle=0$ ) except for the sites in a region $R$, which can be in an arbitrary initial state with finite average particle number (in fact, an almost empty region, i.e., $\left\langle\hat{n}_{j}\right\rangle$ sufficiently small, will also suffice). Note that the region $R$ may very well encompass the major part of the lattice. What we are interested in is how fast these bosons will travel into the empty part $G \backslash R$ of the lattice, as a function of the distance

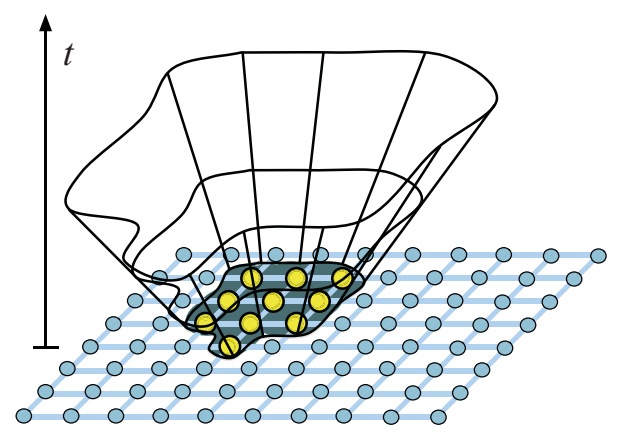

FIG. 1. (Color online) Schematic representation of the "light cone" of particles initially placed into a region $R$ of a lattice (large yellow circles) and then propagating in time $t$ in a way governed by an interacting quantum model, outside of which the influence of these particles is exponentially suppressed. $d(\cdot, \cdot)$ on the underlying graph. In particular, we would like to find a "speed of sound" for the bosons, that is, a velocity $v$ such that for any region $S$ in $G \backslash R$ with $d(S, R) \geqslant l$ [i.e., $d(s, r) \geqslant l \forall s \in S, r \in R]$, and for all times $t$ for which $v t<l$, the expectation value of any observable $\hat{O}_{S}$ on $S$ is equal to the expectation value of the vacuum, up to a correction that decays exponentially away from the light cone, $e^{\gamma(v t-l)}$.

\section{SPEED LIMIT FOR PARTICLES}

To start, we consider the Bose-Hubbard model $\hat{H}_{\mathrm{BH}}$ and focus on measurements of the local particle number operators $\hat{n}_{j}$. This corresponds to looking for bosons at the initially empty sites, and thus captures the most natural notion of particles propagating into a region. Let us denote the initial state by $\rho(0)$, which evolves according to

$$
\dot{\rho}(t)=-i\left[\hat{H}_{\mathrm{BH}}, \rho(t)\right]
$$

for $t \geqslant 0$. Since we are interested in the speed at which particles in the Bose-Hubbard model propagate, let us try to understand how the local particle densities

$$
\alpha_{j}(t)=\operatorname{tr}\left[\hat{n}_{j} \rho(t)\right], \quad j \in G,
$$

evolve under $\hat{H}_{\mathrm{BH}}$. To this end, we derive a bound on the rate at which $\alpha_{j}(\cdot)$ changes, which in turn leads to a bound on the velocity at which particles can propagate through the system. It holds that

$$
\begin{aligned}
\dot{\alpha}_{j}(t) & =-i \operatorname{tr}\left\{\hat{n}_{j}\left[\hat{H}_{\mathrm{BH}}, \rho(t)\right]\right\}=-i \operatorname{tr}\left\{\left[\hat{n}_{j}, \hat{H}_{\mathrm{BH}}\right] \rho(t)\right\} \\
& =2 \tau \sum_{\langle j, k\rangle} \operatorname{Im}\left\{\operatorname{tr}\left[\hat{b}_{k}^{\dagger} \hat{b}_{j} \rho(t)\right]\right\},
\end{aligned}
$$

where the summation runs over all sites $k$ neighboring $j$, $d(j, k)=1$. Since we are only interested in an upper bound on this rate of change, we now consider $\left|\dot{\alpha}_{j}(t)\right|$ and apply the triangle inequality to obtain

$$
\left|\dot{\alpha}_{j}(t)\right| \leqslant 2 \tau \sum_{\langle j, k\rangle}\left|\operatorname{tr}\left[\hat{b}_{k}^{\dagger} \hat{b}_{j} \rho(t)\right]\right| .
$$

To bound this term we use the operator Cauchy-Schwarz inequality, viewing

$$
\operatorname{tr}\left[\hat{b}_{k}^{\dagger} \hat{b}_{j} \rho(t)\right]=\left\langle\hat{b}_{k} \rho^{1 / 2}(t), \hat{b}_{j} \rho^{1 / 2}(t)\right\rangle
$$

as a Hilbert-Schmidt scalar product of $\hat{b}_{j} \rho^{1 / 2}(t)$ and $\hat{b}_{k} \rho^{1 / 2}(t)$, where $\rho^{1 / 2}(t)$ is the matrix square root of $\rho(t)$. This gives rise to

$$
\left|\operatorname{tr}\left[\hat{b}_{k}^{\dagger} \hat{b}_{j} \rho(t)\right]\right| \leqslant\left\{\operatorname{tr}\left[\hat{b}_{k}^{\dagger} \hat{b}_{k} \rho(t)\right] \operatorname{tr}\left[\hat{b}_{j}^{\dagger} \hat{b}_{j} \rho(t)\right]\right\}^{1 / 2} .
$$

Combining this with Eq. (4), we obtain a set of coupled differential inequalities

$$
\left|\dot{\alpha}_{j}(t)\right| \leqslant 2 \tau \sum_{\langle j, k\rangle}\left[\alpha_{j}(t) \alpha_{k}(t)\right]^{1 / 2},
$$

which, using $\sqrt{x y} \leqslant(x+y) / 2$, yields the linearized system

$$
\left|\dot{\alpha}_{j}(t)\right| \leqslant \tau\left[\mathcal{D} \alpha_{j}(t)+\sum_{\langle j, k\rangle} \alpha_{k}(t)\right],
$$

where $\mathcal{D}$ is the maximal vertex degree of the interaction graph. 
We are interested in the worst-case growth of $\alpha_{j}(t)$ as $t$ progresses. This will occur when we have equality in the above expression (i.e., the derivative is as large as possible), and thus a bound $\gamma_{k}(t) \geqslant \alpha_{k}(t)$ is given by the solution of the linear system of differential equations

$$
\dot{\gamma}_{j}(t)=\tau\left[\mathcal{D} \gamma_{j}(t)+\sum_{\langle j, k\rangle} \gamma_{k}(t)\right],
$$

which fulfills $\gamma_{j}(0)=\alpha_{j}(0)$. This solution has the form

$$
\vec{\gamma}(t)=e^{\mathcal{D} \tau t} e^{\tau M t} \vec{\gamma}(0),
$$

where $M$ is the adjacency matrix of the lattice, i.e., $M_{j, k}=1$ if $d(j, k)=1$ and 0 otherwise, and $\vec{\gamma}:=\left(\gamma_{k}\right)_{k \in L}$. This yields an upper bound

$$
\vec{\alpha}(t) \leqslant e^{\mathcal{D} \tau t} e^{\tau M t} \vec{\alpha}(0)
$$

for the expected particle number at time $t$ for any site, for $\vec{\alpha}:=\left(\alpha_{k}\right)_{k \in L}$.

To understand how quickly particles propagate from the initially occupied region $R$ into a region $S$ with $d(R, S) \geqslant$ $l$, we need to consider the off-diagonal block of $e^{\mathcal{D} \tau t} e^{\tau M t}$ corresponding to those two regions. Thus, to obtain a light cone with an exponential decay $\exp (v t-l)$ outside it, we need to understand how rapidly the off-diagonal elements of the banded matrix $M$ grow under exponentiation $e^{\tau M t}$. This can be done by applying theorem 6 from Ref. [10], which yields for the $(i, j)$ th element of $\exp (\tau M t)$ the bound

$$
[\exp (\tau M t)]_{i, j} \leqslant C e^{v_{0} t-d(i, j)}
$$

with velocity $v_{0}=\chi \Delta \tau$, where $\chi \approx 3.59$ is the solution of $\chi \ln \chi=\chi+1, \Delta=\|M\|_{\infty} / 2$ depends on the lattice dimension, and $C=2 \chi^{2} /(\chi-1) \approx 10$. Together with the prefactor $\exp (\mathcal{D} \tau t)$, this gives a Lieb-Robinson velocity $v=v_{0}+\mathcal{D} \tau$. (Note that better bounds can be obtained, cf. [11].) For the scenario of an empty lattice with particles initially placed in a region $R$, this implies that for any $j$ with $d(j, R) \geqslant l$,

$$
\alpha_{j}(t) \leqslant C e^{v t-l} \sum_{k \in R} \alpha_{k}(0)=C N_{0} e^{v t-l},
$$

i.e., up to an exponentially small tail, the particles propagate with a speed no faster than $v$, independent of their initial state. Here, $N_{0}=\sum_{k \in R} \alpha_{k}(0)=\langle\hat{N}\rangle$ is the total number of particles in the system (i.e., the expectation value of the total particle number operator $\hat{N}=\sum_{j} \hat{n}_{j}$ ). Note that while this (unsurprisingly) means that the strength of the signal observed may depend on the number of bosons initially put into the system, the maximum propagation speed $v$ does not depend on $N_{0}$. In fact, for a purely harmonic one-dimensional model for $U=0$, the exact speed of sound is indeed linear in $\tau$, so the above bound is tight up to a small constant prefactor. Also, note that the region $G \backslash R$ need not be strictly empty, as long as its total occupancy is smaller than the propagation effect we want to observe. What we have derived is in fact a bound on how fast particles can propagate from any region into any other region.

\section{SPEED LIMIT FOR GENERAL OBSERVABLES}

Having understood how to obtain a bound on the propagation speed of particles, we now turn to more general observables. First, let us show how we can bound the higher moments of the particle number operator. For $p \geqslant 1$,

$$
\begin{aligned}
\alpha_{j}^{(p)}(t) & =\operatorname{tr}\left[\hat{n}_{j}^{p} \rho(t)\right] \\
& =\sum_{N} \operatorname{tr}\left[\hat{n}_{j} \hat{n}_{j}^{p-1} P_{N} \rho(t) P_{N}\right] \\
& \leqslant \sum_{N} \operatorname{tr}\left[\hat{n}_{j} N^{p-1} P_{N} \rho(t) P_{N}\right] \\
& \stackrel{(7)}{\leqslant} \sum_{N} N^{p-1}\left[C N e^{v t-l}\right] \operatorname{tr}[\rho(t)] \\
& =C\left\langle\hat{N}^{p}\right\rangle e^{v t-l},
\end{aligned}
$$

where $P_{N}$ projects onto the subspace with a total of $N$ particles, and we have used that Eq. (7) applies to each subspace with fixed particle number independently as the Hamiltonian commutes with $P_{N}$. Here, $\left\langle\hat{N}^{p}\right\rangle$ denotes the (time-independent) expectation value of the $p$ th moment of the total particle number operator. This proves a Lieb-Robinson bound for the higher moments of the particle number operator.

Let us now turn our attention toward arbitrary local observables $\hat{A}_{j}$. Any such observable can be written as $\hat{A}_{j}=\sum_{p, q} c_{p, q}\left(\hat{b}_{j}^{\dagger}\right)^{p} \hat{b}_{j}^{q}$, and we thus have that

$$
\begin{aligned}
\left|\operatorname{tr}\left[\hat{A}_{j} \rho(t)\right]\right| & \leqslant \sum_{p, q}\left|c_{p, q}\right|\left|\operatorname{tr}\left[\left(\hat{b}_{j}^{\dagger}\right)^{p} \hat{b}_{j}^{q} \rho(t)\right]\right| \\
& \leqslant \sum_{p, q}\left|c_{p, q}\right|\left\{\operatorname{tr}\left[\left(\hat{b}_{j}^{\dagger}\right)^{p} \hat{b}_{j}^{p} \rho(t)\right] \operatorname{tr}\left[\left(\hat{b}_{j}^{\dagger}\right)^{q} \hat{b}_{j}^{q} \rho(t)\right]\right\}^{1 / 2} .
\end{aligned}
$$

In turn, for $p>0$,

$$
\begin{aligned}
\operatorname{tr}\left[\left(\hat{b}_{j}^{\dagger}\right)^{p} \hat{b}_{j}^{p} \rho(t)\right] & =\operatorname{tr}\left[\hat{n}_{j}\left(\hat{n}_{j}-1\right) \cdots\left(\hat{n}_{j}-p+1\right) \rho(t)\right] \\
& =\sum_{r=1}^{p} d_{r, p} \alpha_{j}^{(r)}(t) \leqslant \tilde{C}_{p} e^{v t-l}
\end{aligned}
$$

by virtue of Eq. (7), for some constant $\tilde{C}_{p}$. If $p=0$, we trivially have $\operatorname{tr}[\rho(t)]=1$. Together, this yields a bound

$$
\left|\operatorname{tr}\left[\hat{A}_{j} \rho(t)\right]\right| \leqslant C^{\prime} e^{v t-l}
$$

if $c_{0, q}=c_{p, 0}=0$ for all $p$ and $q$, and

$$
\left|\operatorname{tr}\left[\hat{A}_{j} \rho(t)\right]\right| \leqslant C^{\prime} e^{(v t-l) / 2}
$$

otherwise, where we have assumed that $\sum\left|c_{p, q}\right|$ is finite, and used that without loss of generality $c_{0,0}=0$. In both cases, this means that outside the light cone given by $v t=l, \operatorname{tr}\left[\hat{A}_{j} \rho(t)\right]$ decays exponentially; however, the decay is on double the length scale in the latter case. 
Finally, observables acting on more than one site can be bounded analogously to the local case: any two-site operator acting on sites $j, k$ can be written as the sum of terms $\hat{A}_{j} \hat{A}_{k}$, and

$$
\left|\operatorname{tr}\left[\hat{A}_{j} \hat{A}_{k} \rho(t)\right]\right| \leqslant\left\{\operatorname{tr}\left[\hat{A}_{j}^{\dagger} \hat{A}_{j} \rho(t)\right] \operatorname{tr}\left[\hat{A}_{k} \hat{A}_{k}^{\dagger} \rho(t)\right]\right\}^{1 / 2} .
$$

The terms on the right-hand side are local observables that can be bounded as before by $\exp (v t-l)$, yielding the same exponential bound for two-site-and recursively for manysite-observables. Note that cases exist where terms that are bounded by $\exp [(v t-l) / 2]$ only appear, and in addition one of the $\hat{A}$ 's above could be the identity. Thus, bounds of the form $\exp [(v t-l) / \kappa]$ can occur, where $\kappa$ can grow exponentially in the block size. This, however, still implies that the signal is exponentially small outside the light cone.

\section{GENERALIZATIONS}

While we have illustrated our arguments for the BoseHubbard model, they generalize straightforwardly to the more general class of models described by Eq. (2). First, it is clear that we can replace the on-site repulsion and chemical potential in the Bose-Hubbard model by any type of interaction (even a nonlocal one) that only depends on the particle numbers, since any such term vanishes in the commutator $\left[\hat{n}_{j}, \hat{H}\right]$ in Eq. (3). Second, for systems that contain several types of bosons the same arguments apply. Such systems can be modeled using multiple copies of the original graph, each of which supports the hopping of one individual boson species, and one obtains independent differential inequalities for the particle densities $\alpha_{j, s}(t)=\operatorname{tr}\left[\hat{n}_{j, s} \rho(t)\right]$ for each species.

Beyond general bosonic models, our arguments also apply to fermions and mixtures of bosons and fermions [12], and in fact even to anyonic systems. Again, in a first step one can decouple the individual species of particles (which mutually commute) to hop on independent graphs. Then, it is easy to check that our arguments work independently of the statistics of the particles, since $\left[\hat{n}_{j}, \hat{H}\right]$ in Eq. (3) evaluates to the same expression in terms of the fermionic (anyonic) creation and annihilation operators. Even better, fermionic and anyonic systems yield stronger bounds for the higher moments, and thus for the scenario of general local observables. In Eq. (8), $\hat{n}_{j}^{p-1}$ can be bounded by 1 instead of $\hat{N}^{p-1}$, which yields a bound $\alpha_{j}^{(p)}(t) \leqslant C N_{0} e^{v t-l}$ on the higher moments. Corresponding results also follow for spin systems, since these can be described as hard-core bosons.

Our arguments work not only for unitary theories, but also for certain types of dissipative (Markovian) models, extending the work of Ref. [13] to bosonic systems. For instance, in the practically relevant case of a bosonic system with particle losses, we have that

$$
\dot{\rho}(t)=-i\left[\hat{H}_{\mathrm{BH}}, \rho\right]-\lambda \sum_{j}\left[\left\{\hat{b}_{j}^{\dagger} \hat{b}_{j}, \rho(t)\right\}-2 \hat{b}_{j} \rho(t) \hat{b}_{j}^{\dagger}\right] .
$$

Therefore,

$$
\dot{\alpha}_{j}(t)=-i \operatorname{tr}\left\{\left[\hat{n}_{j}, \hat{H}_{\mathrm{BH}}\right] \rho(t)\right\}-\lambda \operatorname{tr}\left[\hat{n}_{j} \rho(t)\right],
$$

which shows that the contribution from the dissipative term to $\dot{\alpha}_{j}$ is negative; thus tighter differential inequalities and a lower speed of sound than in the Hamiltonian case can be obtained.

\section{SUMMARY}

In summary, we have proven that there is a maximum speed at which information in systems of interacting particles can propagate under certain initial conditions, and in particular, to the speed at which interacting bosons can propagate into any initially empty region of the system, such as when released from a trap. This is the first speed limit for the propagation of information in systems of strongly interacting bosons, a scenario that cannot be assessed using the established techniques of Lieb-Robinson bounds due to the unboundedness of the bosonic hopping operator. Our argument applies equally to bosonic, fermionic, anyonic, and spin systems, as well as mixtures thereof, even with interaction terms between different particle types, and can be generalized to also address systems with dissipation.

The idea of studying information propagation by restricting to a specific set of observables and investigating the resulting worst-case differential equation can also be applied to the study of continuous systems. This can be done either by taking an appropriate continuum limit of a lattice model, or by directly considering a corresponding differential equation for the particle density that is continuous in space.

One setting where our approach seems to fail is that of systems with a constant initial density of particles, e.g., a "Mott state" with $m$ particles per site. This invalidates our assumption that there is a region without particles. However, it seems intuitively plausible that an information-propagation bound should still apply because it should take some time for an initial disturbance, caused, e.g., by the addition of a particle at some site, to move through the system. Unfortunately our proof technique does not directly apply to this case. To illustrate why this might be a complex problem consider the example of the Bose-Hubbard model with a negative $U$. In this case it is energetically favorable for particles to "clump together.'. With an initial state given by the Mott state with $m$ particles per site it seems likely the system will be unstable to perturbations, and little towers of clumped particles could accelerate through the system. Any general proof technique for information-propagation bounds will need to be able to cope with this situation, or at least provide some nontrivial criteria for models to obey such bounds.

\section{ACKNOWLEDGMENTS}

This work was supported by the EU (COMPAS, MINOS, QESSENCE), the EURYI, the BMBF (QuOReP), the Gordon and Betty Moore Foundation through Caltech's Center for the Physics of Information, the National Science Foundation under Grant No. PHY-0803371, and the ARO under Grant No. W911NF-09-1-0442. Part of this work was done at the Mittag-Leffler-Institute. 
[1] E. H. Lieb and D. W. Robinson, Commun. Math. Phys. 28, 251 (1972).

[2] M. B. Hastings, Phys. Rev. B 69, 104431 (2004); B. Nachtergaele and R. Sims, Commun. Math. Phys. 265, 119 (2006).

[3] B. Nachtergaele and R. Sims, e-print arXiv:1004.2086.

[4] J. Eisert and T. J. Osborne, Phys. Rev. Lett. 97, 150404 (2006); S. Bravyi, M. B. Hastings, and F. Verstraete, ibid. 97, 050401 (2006); C. K. Burrell and T. J. Osborne, ibid. 99, 167201 (2007); A. Hamma, F. Markopoulou, I. Premont-Schwarz, and S. Severini, ibid. 102, 017204 (2009); J. Eisert, M. Cramer, and M. B. Plenio, Rev. Mod. Phys. 82, 277 (2010).

[5] J. Eisert and D. Gross, Phys. Rev. Lett. 102, 240501 (2009).

[6] T. J. Osborne, Phys. Rev. Lett. 97, 157202 (2006); M. B. Hastings, Phys. Rev. B 77, 144302 (2008).

[7] I. Bloch, J. Dalibard, and W. Zwerger, Rev. Mod. Phys. 80, 885 (2008); S. Trotzky et al., Nature Physics (in press), e-print arXiv:1101.2659.

[8] L. Hackermüller et al., Science 327, 1621 (2010).

[9] B. Nachtergaele et al., Commun. Math. Phys. 286, 1073 (2009); Rev. Math. Phys. 22, 207 (2010).

[10] M. Cramer and J. Eisert, New J. Phys. 8, 71 (2006).

[11] There are two ways to obtain better bounds on the velocity. First, we can bound $\sqrt{x y} \leqslant \frac{1}{2}(\lambda x+y / \lambda)$, which gives a velocity bound $\lambda v_{0}+\mathcal{D} \tau / \lambda$ for any $\lambda>0$. Second, we can solve the nonlinear differential inequality (5) by substituting $\alpha_{j}(t)=$ : $\beta_{j}(t)^{2}$, which gives linear inequalities

$$
\dot{\beta}_{j}(t) \leqslant \tau \sum_{\langle j, k\rangle} \beta_{k}(t),
$$

with initial conditions $\beta(0)=\sqrt{\alpha(0)}$. The worst-case solution of this system is $\vec{\beta}(t)=e^{\tau M t} \vec{\beta}(0)$, which, using the previous estimate of $e^{\tau M t}$, yields a velocity $v_{0}$. To obtain bounds on $\alpha_{j}(t)$, we need to square this bound. On the one hand, this implies that the correlations outside the light cone decay as $e^{2(v t-l)}$; however, it also yields an unfavorable dependence of the prefactor on the initial conditions, $\left[\sum_{j} \sqrt{\alpha_{j}(0)}\right]^{2}$, which can diverge for a fixed number of bosons as the region $R$ grows.

[12] A. Albus, F. Illuminati, and J. Eisert, Phys. Rev. A 68, 023606 (2003); H. P. Büchler and G. Blatter, ibid. 69, 063603 (2004); M. Cramer, J. Eisert, and F. Illuminati, Phys. Rev. Lett. 93, 190405 (2004); M. Lewenstein, L. Santos, M. A. Baranov, and H. Fehrmann, ibid. 92, 050401 (2004).

[13] D. Poulin, Phys. Rev. Lett. 104, 190401 (2010); C. K. Burrell, J. Eisert, and T. J. Osborne, Phys. Rev. A 80, 052319 (2009). 